\begin{tabular}{|c|l|}
\hline Title & $\begin{array}{l}\text { Laser trapping and picosecond time resolved spectroscopy of water droplets in air : cavity-enhanced spontaneous } \\
\text { emission of Ru(bpy)3CI2 }\end{array}$ \\
\hline Author(s) & Ishizaka, Shoji; Suzuki, Y uya; Kitamura, Noboru \\
\hline Citation & $\begin{array}{l}\text { Physical Chemistry Chemical Physics, 12(33), 9852-9857 } \\
\text { https://doi.org/_0.1039/c003887c }\end{array}$ \\
\hline Issue Date & 2010-09-07 \\
\hline Doc URL & http://hdl.handle.net/2115/45566 \\
\hline Rights & Phys. Chem. Chem. Phys., 2010, 12, 9852-9857- Reproduced by permission of the PCCP Owner Societies \\
\hline Type & article (author version) \\
\hline File Information & PCcP12-33_9852-9857.pdf \\
\hline
\end{tabular}

Instructions for use 


\section{Laser trapping and picosecond time-resolved spectroscopy of water droplets in air: cavity-enhanced spontaneous emission of $\mathrm{Ru}(\mathrm{bpy})_{3} \mathrm{Cl}_{2}$}

Shoji Ishizaka*, Yuya Suzuki, and Noboru Kitamura*

Department of Chemistry, Graduate School of Science, Hokkaido University, Kita-ku, Sapporo 060-0810.

*Corresponding authors.

e-mail address: shoji@sci.hokudai.ac.jp (S. Ishizaka),kitamura@sci.hokudai.ac.jp (N. Kitamura). 


\section{Abstract}

Whispering gallery mode (WGM) resonances were observed in the emission spectrum of $\mathrm{Ru}(\mathrm{bpy})_{3}{ }^{2+}$ (bpy $=2,2^{\prime}$-bipyridine) in a single laser-trapped water droplet levitated in air. The emission decay profiles of $\mathrm{Ru}(\mathrm{bpy})_{3}{ }^{2+}$ in the water droplets comprised fast and slow decay components. The emission lifetime of the slow decay component was independent of the diameter of the droplet, and corresponded to the value in a bulk aqueous solution. On the other hand, the emission lifetime of the fast decay component decreased with decreasing the droplet diameter, which could be ascribed to the cavity-enhanced spontaneous emission. The decrease in the emission lifetime of the fast decay component as a function of the droplet diameter was explained on the basis of cavity quantum electrodynamic (QED) effects. It was shown that the mode characteristic of WGM resonances and the enhancement factor of the radiative rate of $\mathrm{Ru}(\mathrm{bpy})_{3}{ }^{2+}$ were controlled by the size of the water droplet. 


\section{Introduction}

Noncontact levitation of a single micrometer-sized water droplet in air can be achieved

by a laser trapping technique and, therefore, the laser trapping technique is a powerful means to study the aerosol chemistry. ${ }^{1-12}$ Since the refractive index $(m)$ of water is higher than that of air, the dye emission radiated from the inside of the droplet is reflected totally at the droplet/air boundary and propagates circumferentially to produce standing waves at the boundary. This phenomenon is called whispering gallery mode (WGM) resonances and the emission is amplified by the WGM resonances in a water droplet. ${ }^{3}$ Thus, a dye solubilized in micrometer-sized water droplets shows optical and spectroscopic properties different from those in bulk water. Furthermore, the cavity quantum electrodynamic (QED) theory predicts that spontaneous emission from an atom or molecule is enhanced in a spherical microcavity under the conditions that the emission wavelengths coincide with the cavity resonance wavelengths. ${ }^{13-19}$ Such effects were first discussed by Purcell, who noted that the changes in the density of the final state per unit volume and unit frequency would enhance greatly a spontaneous emission probability as compared with that normally observed in free space. ${ }^{20}$ Controls of the spontaneous and stimulated emission properties of a dye confined in a spherical microcavity have been of considerable interest due to its applications to low threshold lasing, ${ }^{21}$ single molecular detection, ${ }^{22}$ and enhanced energy transfer. ${ }^{23,24}$ 
The prerequisite condition to observe the radiative rate enhancement due to the cavity QED effects is the presence of WGM resonances in the fluorescence spectrum. However, the number of the report on simultaneous observations of both WGM resonances in the spectrum and radiative rate enhancement of a fluorophore in a spherical microcavity are very few. Sandeep and Bisht reported the emission spectra and emission decay profiles of 9-amino acridine hydrochloride hydrate (9AAHH) doped in polymethyl methacrylate (PMMA) microspheres and, demonstrated that observations of WGM resonances in the spectra and radiative rate enhancement of $9 \mathrm{AAHH} .{ }^{16-18}$ To the best of our knowledge, this is the only one system showing simultaneous WGM resonances and radiative rate enhancement. However, it has been reported that the enhancement factor of the radiative rate of 9AAHH is strongly depended on the concentration of the dye, because of re-absorption of the fluorescence by the ground state dye in the microsphere. ${ }^{17,18}$ The theoretical model for the size dependence of the emission lifetime of a dye in a microsphere has been proposed by Arnold: a three dimensional spherical cavity model. ${ }^{25}$ Nonetheless, an applicability of the theory has been discussed only for radiative rate enhancement from the singlet-excited state of a dye in a microsphere. ${ }^{16}$ We think that further detailed investigations are necessary to understand such phenomena.

In this study, we conducted picosecond time-resolved spectroscopy of a dye dissolved in micrometer-sized water droplets in air by means of a laser trapping technique. We 
employed tris(2,2'-bipyridine)ruthenium(II) dichloride hexahydrate $\left(\mathrm{Ru}(\mathrm{bpy})_{3}{ }^{2+}\right)$ as a dye. Since the emissive excited state of the complex is the metal-to-ligand charge transfer (MLCT) excited triplet state, there is almost no overlap between the absorption and emission (i.e., phosphorescence) spectra. WGM resonances are strongly affected by the overlap between the absorption and emission spectra of a dye owing to re-absorption of the emission by the dye molecules in the ground-state. Therefore, the phosphorescence emission of $\mathrm{Ru}(\mathrm{bpy})_{3}{ }^{2+}$ is very suitable for observation of the WGM resonances in water droplets. In this study, we demonstrate simultaneous observations of WGM resonances in the emission spectrum and radiative rate enhancement of $\mathrm{Ru}(\mathrm{bpy})_{3}{ }^{2+}$ dissolved in single aerosol water droplets.

\section{Experimental}

Water was purified by distillation and deionization prior to use (GSR-200, Advantec Toyo Co., Ltd.). Tris(2,2'-bipyridine)ruthenium(II) dichloride hexahydrate (Sigma-Aldrich Co.) and sodium chloride (Wako Pure Chemical Co. Ltd., analytical grade) were used without further purification.

A schematic illustration of the experimental setup is shown in Fig. 1. Aerosol water droplets containing $\mathrm{Ru}(\mathrm{bpy})_{3}{ }^{2+}\left(10 \mathrm{mmol} / \mathrm{dm}^{3}\right)$ and sodium chloride $\left(1.0 \mathrm{~mol} / \mathrm{dm}^{3}\right)$ were generated by an ultrasonic nebuliser (Omron, NE-U07) and introduced into a chamber set 
on the stage of an inverted optical microscope (Nikon, Eclipse TE300). The presence of $\mathrm{NaCl}$ reduces the vapor pressure of the droplet, allowing the droplet to be retained for a long time in the optical trap under saturated relative humidity. Among water droplets, single water droplets were trapped by a focused $1064 \mathrm{~nm}$ laser beam from a CW Nd:YAG laser (Spectron, SL-902T) introduced to the microscope through an oil immersion objective lens $(\times 100, \mathrm{NA}=1.30)$. $\mathrm{Ru}(\mathrm{bpy})_{3}{ }^{2+}$ in the water droplets was excited by femtosecond laser pulses from a Ti-Sapphire-OPA laser (Coherent, $480 \mathrm{~nm}$, repetition rate $=100 \mathrm{kHz}, \mathrm{FWHM}=200 \mathrm{fs}$ ), which were introduced into the microscope coaxially with the trapped laser beam. Emission from the dye in the trapped water droplet was collected by the same objective lens and analyzed by a photodecector system. Emission spectra were measured by a cooled CCD detector (Andor Tech, DV420-BV) equipped with a polychromator (150 grooves/mm). Emission decay profiles at $620 \pm 6 \mathrm{~nm}$ were measured by using a microchannel-plate photomultiplier tube (Hamamatsu, R3809U-50), instead of using the cooled CCD detector described above, and analyzed by a single-photon counting module (Edinburgh Instruments, SPC-300). Emission decay curves were analyzed by a non-linear least-squares iterative convolution method based on the Marquardt algorithm. ${ }^{26}$ All measurements were conducted at room temperature. 


\section{Results and Discussion}

WGM emission resonances of Ru(bpy) ${ }_{3}{ }^{2+}$ in laser-trapped single aerosol water droplets.

Single micrometer-sized water droplets were trapped from a dense flow of aerosol water droplets (averaged diameter $\sim 2 \mu \mathrm{m}$ ) introduced into the chamber as shown in Fig. 2. During laser trapping, the droplet coalesced with other non-trapped aerosol water droplets and grew gradually in the size. The size of the trapped water droplet could be controlled from 2 to $15 \mu \mathrm{m}$ in diameter. Single water droplets could be levitated stably in several hours. By using this technique, we conducted spectroscopic measurements of single aerosol water droplets.

Emission spectra of Ru(bpy) ${ }_{3}{ }^{2+}$ in a bulk aqueous solution and single aerosol water droplets are shown in Fig. 3. The emission spectrum observed for a bulk aqueous solution was broad and structureless (Fig. 3(a)), while that observed for aerosol water droplets exhibited many sharp peaks superimposed on the broad emission band of Ru(bpy) ${ }^{2+}$ as shown in Fig. 3(b) (d). The spacing between mutually adjacent peaks was larger for a smaller droplet, and the observed peak number increased with an increase in the droplet size. Since the WGM resonances in the droplet occur at certain wavelengths for a given droplet size, the distinct resonance peaks should be observed from the aerosol water droplets. Theoretically, the WGM resonances can be analyzed by the Mie scattering 
theory. ${ }^{27}$ The scattering efficiency $\left(Q_{s}\right)$ of a microsphere is given by eqn (1),

$$
Q_{s}=\frac{2}{x^{2}} \sum_{n=1}^{\infty}(2 n+1)\left(\left|a_{n, l}\right|^{2}+\left|b_{n, l}\right|^{2}\right)
$$

where $x=2 \pi r / \lambda$ is known as the size parameter. Here, $\lambda$ is the wavelength of scattered light and $r$ is the radius of the microsphere. The coefficient $a_{n, l}$ and $b_{n, l}$ are the spherical Bessel $\left[j_{\mathrm{n}}(x)\right]$ and Hankel $\left[h_{\mathrm{n}}{ }^{(l)}(x)\right]$ functions of the first kind and are given by eqns (2) and (3), respectively,

$$
\begin{gathered}
a_{n, l}=\frac{j_{n}(x)\left[m x j_{n}(m x)\right]-m^{2} j_{n}(m x)\left[x j_{n}(x)\right]}{h_{n}^{(l)}(x)\left[m x j_{n}(m x)\right]-m^{2} j_{n}(m x)\left[x h_{n}^{(l)}(x)\right]} \\
b_{n, l}=\frac{j_{n}(x)\left[m x j_{n}(m x)\right]-j_{n}(m x)\left[x j_{n}(x)\right]}{h_{n}^{(l)}(x)\left[m x j_{n}(m x)\right]-j_{n}(m x)\left[x h_{n}^{(l)}(x)\right]}
\end{gathered}
$$

where $m$ represents the refractive index of the microsphere. The integer $n$ denotes the order of the spherical Bessel and Hankel functions describing the radial field distribution and is known as the angular mode number. Here, $n$ and $l$ represent the mode number and mode order of a resonance, respectively. As shown in eqns (1) (3), the Mie scattering efficiency and WGM resonances depend on the radius and refractive index of a droplet. Fig. 4(a) shows the emission spectrum of $\mathrm{Ru}(\mathrm{bpy})_{3}{ }^{2+}$ in a water droplet with the radius of $\sim 3.5 \mu \mathrm{m}$. Fig. 4(b) shows the theoretical simulation of the scattering efficiency $\left(Q_{\mathrm{s}}\right)$ of a droplet with the refractive index of 1.343 and the radius of $3.373 \mu \mathrm{m} .{ }^{28}$ Dashed lines are guides to eyes. It is worth noting that the peak positions of $Q_{\mathrm{s}}$ match very well with observed peaks in the emission spectrum. These results demonstrate that the observed 
sharp peaks in the emission spectrum are ascribed essentially to the WGM resonances.

Size dependence of emission lifetime of $\mathrm{Ru}(\mathrm{bpy})_{3}{ }^{2+}$ in aerosol water droplets. The observation of the WGM resonances in the emission spectra of Ru(bpy) ${ }_{3}^{2+}$ in the aerosol water droplets suggests that the radiative rate constant of the complex is also influenced by the light resonances and, thus, a change in the emission lifetime. To confirm this, we conducted emission lifetime measurements of Ru(bpy) ${ }_{3}{ }^{2+}$ in aerosol water droplets. Fig. 5 shows the typical emission decay profiles of $\mathrm{Ru}(\mathrm{bpy})_{3}{ }^{2+}$ in a bulk aqueous solution and aerosol water droplets. Fitting curves are shown as the solid curves in Fig. 5, and the fitting parameters are summarized in Table 1. The emission decay profile of $\mathrm{Ru}(\mathrm{bpy})_{3}{ }^{2+}$ in a bulk aqueous solution was fitted satisfactorily by a single exponential function with the time constant of 403 ns (Fig. 5(a)). On the other hand, the emission decay profiles observed for the aerosol water droplets were best fitted by double exponential functions as judged by the relevant $\chi^{2}$ and Durbin-Watson (DW) parameters for the fittings as shown in Table 1. Within an experimental error, the long lifetime component $\left(\tau_{2}\right)$ observed from each droplet agreed with that in the bulk aqueous solution: $403 \pm 1$ ns. On the other hand, the time constant of the short lifetime component $\left(\tau_{1}\right)$ decreased from $256 \pm 5.6$ to $28.8 \pm 0.2$ ns with a decrease in the droplet diameter from 6.0 to $3.0 \mu \mathrm{m}$ as shown in Table 1. 
In this study, a $1064 \mathrm{~nm}$ laser beam was used for the trapping light source of aerosol water droplets. Water absorbs $1064 \mathrm{~nm}$ light, and photo-thermal local heating $(\Delta T)$ of a water droplet by $1064 \mathrm{~nm}$ light absorption can be roughly estimated by the following equation, 29

$$
\Delta T=\frac{Q}{4 \pi \kappa r}
$$

where $\kappa$ and $r$ represent the thermal conductivity $\left(0.6 \mathrm{~W} \mathrm{~m}^{-1} \mathrm{~K}^{-1}\right)$ and radius of a water droplet, respectively. Typical laser power of a $1064 \mathrm{~nm}$ laser beam $\left(P_{1064}\right)$ used for trapping of a water droplet $(r=2.5 \mu \mathrm{m})$ is $\sim 10 \mathrm{~mW}$. The conversion of the photon energy to heat (Q) of a $1064 \mathrm{~nm}$ laser beam absorbed by the water droplet was estimated based on the absorption of $\mathrm{H}_{2} \mathrm{O}$ at $1064 \mathrm{~nm}$ (absorbance $=2.8 \times 10^{-5}$ for $5 \mu \mathrm{m}$ optical path length) and $P_{1064}$. At $P_{1064}=10 \mathrm{~mW}$, the relevant temperature increase in the droplet $(\Delta T)$ can be calculated to be $\sim 0.03^{\circ} \mathrm{C}$, and is too small to explain enhancement of the transition rate of the emission from $\mathrm{Ru}(\mathrm{bpy})_{3}{ }^{2+}$ in the droplet. Therefore, the presence of the short lifetime component $\left(\tau_{1}\right)$ observed for the droplet cannot be ascribed to the photo-thermal local heating of water droplets. Furthermore, we confirmed that the time constants of the short lifetime component $\left(\tau_{1}\right)$ of $\mathrm{Ru}(\mathrm{bpy})_{3}{ }^{2+}$ in the droplets were independent of excitation laser power, as shown in Fig. 6 and Table 2. Therefore, the $\tau_{1}$ component observed for the droplet is caused by neither the stimulated emission processes, excited triplet-triplet states annihilation, nor photo-thermal effects. Therefore, 
we concluded that the transition rate of spontaneous emission in the water droplet is essentially different from that in the bulk medium. One of the necessary conditions to observe radiative rate enhancement due to the cavity QED is the presence of WGM resonances at the droplet/air boundary. At the droplet/air boundary, electronic coupling of the emission transition with the WGM modes causes the increase in the final state density of $\mathrm{Ru}(\mathrm{bpy})_{3}{ }^{2+}$ and this should give rise to the enhancement of the radiative transition rate as predicted by the Fermi's golden rule. In the center of the droplet, on the other hand, the dye is unaffected by the whispering gallery modes so that the emission decay rate is identical to that in a bulk medium. These are the reasons for the short $\left(\tau_{1}\right)$ and long lifetime $\left(\tau_{2}\right)$ components observed from the Ru(bpy) ${ }_{3}{ }^{2+} /$ water droplets.

The size dependence of the fluorescence lifetime of a dye in micrometer-sized particles has been discussed on the basis of the theory proposed by Yokoyama and Brorson. ${ }^{30}$ On the assumption that enhanced spontaneous emission is caused by light resonances in a one-dimensional microcavity, they have predicted that the rate constant of spontaneous emission should proportional to an inverse of a sphere radius: $1 / r$ hypothesis model. The enhancement factor ( $\xi$ ) of spontaneous emission is defined as the ratio of the emission lifetime observed in a bulk system to that observed in a microsphere. The size dependence of $\xi$ can be then analyzed by eqn (5),

$$
\xi=\frac{\Delta_{c}}{\Gamma_{h}}
$$


where $\Gamma_{h}$ is the homogeneous linewidth of a dye molecule and, $\Delta_{c}$ represents the cavity mode spacing as given in eqn (6).

$$
\Delta_{c}=\frac{\tan ^{-1}\left(m^{2}-1\right)^{1 / 2}}{2 \pi r\left(m^{2}-1\right)^{1 / 2}}
$$

For the present Ru(bpy) ${ }_{3}{ }^{2+} /$ water droplet system, the relationship between $\xi$ and the droplet diameter is shown by the filled boxes in Fig. 7. The enhancement factor increases sharply when the droplet diameter becomes smaller than $5 \mu \mathrm{m}$. The result of the non-linear least means square fitting by eqn (5) $\left(\Gamma_{h}=82 \mathrm{~cm}^{-1}\right)$ is shown by the dashed line in Fig. 7. It is very clear from Fig. 7 that the experimental data increase more rapidly with decreasing the size than $1 / r$. Similar discrepancy between observed data and those predicted from the model has been also reported for the size dependence of the fluorescence lifetime of Rhodamine 6G (R6G) in glycerol droplets levitated in air by means of an electrodynamic trap. ${ }^{25}$ Such discrepancies are suggested to come from the assumption of the single dimensional cavity model in the theory. On the other hand, the cavity QED theory considering 3-dimensional nature of the cavity has been proposed by Arnold and, in this model, $\xi$ is given by the following equation, ${ }^{25}$

$$
\xi=1+\frac{f}{r}\left(\frac{2 c}{\pi m \Gamma_{h}} \cdot \frac{1}{r}-1\right)
$$

where $c / m$ is the velocity of light in the medium and, $f$ is related to the degeneracy $(D)$ of the WGM modes and the droplet volume $\left(V_{\mathrm{p}}\right)$ as $f / m=D / \rho V_{\mathrm{p}} \Delta_{c}$. The degeneracy of the 
WGM modes is given by $2 n+1$, where $n$ is the corresponding mode number. According to this theory, the enhance factor determined by the present experiments almost agrees with the theoretical curve $\left(\Gamma_{h}=210 \mathrm{~cm}^{-1}\right.$ and $\left.f=10 \mu \mathrm{m}\right)$ as shown by the solid curve in Fig. 7 . Therefore, we conclude that the 3-dimensional cavity model in eqn (7) explain very well the experimental data as compared with the simple $1 / r$ model in eqn (5). To date, an applicability of the 3-dimensional cavity model (eqn (7)) has been discussed only for radiative rate enhancement of the excited singlet state of a dye in microspheres, i.e. R6G in glycerol droplets ${ }^{25}$ or 9AAHH in PMMA microspheres. ${ }^{16}$ In both cases, the enhancement factors observed for the fluorescence might be affected by re-absorption of the dye fluorescence by the ground-state dye, which would reduce considerably radiative rate enhancement. On the other hand, since the emission from $\mathrm{Ru}(\mathrm{bpy})_{3}{ }^{2+}$ is originated from the MLCT excited triplet state, there is no spectral overlap between the absorption and emission spectra and, therefore, radiative rate enhancement shown in Fig. 7 is free from the re-absorption effects of the dye emission by the ground-state dye. In practice, WGM resonances were clearly observed in the whole spectral region of the emission spectrum as shown in Fig. 3. Therefore, we conclude that the change in the spontaneous emission rate of $\mathrm{Ru}(\mathrm{bpy})_{3}{ }^{2+}$ is caused essentially by the cavity QED effects and the results are well explained by the 3-dimensional cavity model in eqn (7). 


\section{Conclusions}

We succeeded in laser trapping-spectroscopy of single micrometer-sized water droplets in air. WGM resonances and radiative rate enhancement of Ru(bpy) ${ }^{2+}$ could be observed simultaneously in an aerosol water droplet. As demonstrated in the present study, the photophysical properties of $\mathrm{Ru}(\mathrm{bpy})_{3}{ }^{2+}$ in aerosol water droplets are very characteristic owing to the microcavity effects of the droplet. However, the nonradiative transition processes of the dye dissolved in a water droplet are still unclear, and a further study on the photophysical characteristics of $\mathrm{Ru}(\mathrm{bpy})_{3}{ }^{2+}$ in aerosol water droplets including the emission quantum yield measurements of the dye are absolutely necessary, which is our next target of the study. We convince that a laser trapping-spectroscopy technique and high sensitive detection of a molecule by using microcavity effects of a water droplet can contribute to the study on the aerosol chemistry.

\section{Acknowledgement}

S.I. is grateful for the Global COE Program (Project No. B01: Catalysis as the Basis for Innovation in Materials Science) for the partial support of the research. N.K. acknowledges the Grant-in-Aids from the Ministry of Education, Culture, Sports, Science, and Technology, Japan (No. 19205010) for the partial support of the research. 


\section{References and Notes}

1 A. Ashkin, Science, 1975, 187, 1073-1075.

2 N. Magome, M. I. Kohira, E. Hayata, S. Mukai and K. Yoshikawa, J. Phys. Chem. B, 2003, 107, 3988-3990.

3 R. Symes, R. M. Sayer and J. P. Reid, Phys. Chem. Chem. Phys., 2004, 6, 474-478.

4 R. J. Hopkins, L. Mitchem, A. D. Ward and J. P. Reid, Phys. Chem. Chem. Phys., 2004, 6, 4924-4927.

5 L. Mitchem and J. P. Reid, Chem. Soc. Rev., 2008, 37, 756-769.

6 K. J. Knox and J. P. Reid, J. Phys. Chem. A, 2008, 112, 10439-10441.

7 M. D. King, K. C. Thompson and A. D. Ward, J. Am. Chem. Soc., 2004, 126, $16710-16711$.

8 M. Guillon, K. Dholakia and D. McGloin, Opt. Express, 2008, 16, 7655-7664.

9 C. Mund and R. Zellner, J. Mol. Struct., 2003, 661-662, 491-500.

10 C. Mund and R. Zellner, ChemPhysChem, 2003, 4, 630-638.

11 C. Mund and R. Zellner, ChemPhysChem, 2003, 4, 638-645.

12 N. Jordanov and R. Zellner, 2006, Phys. Chem. Chem. Phys., 2006, 8, 2579-2764.

13 H-B. Lin, J. D. Eversole, C. D. Merrit and A. J. Campillo, Phys. Rev. A, 1992, 45, $6756-6760$.

14 M. D. Barnes, W. B. Whitten, S. Arnold and J. M. Ramsey, J. Chem. Phys., 1992, 97, $7842-7845$.

15 H. Fujiwara, K. Sasaki and H. Masuhara, J. Appl. Phys., 1999, 85, 2052-2056.

16 P. Sandeep and P. B. Bisht, Chem. Phys. Lett., 2003, 371, 327-332.

17 P. Sandeep and P. B. Bisht, Chem. Phys. Lett., 2005, 415, 15-19.

18 P. Sandeep and P. B. Bisht, J. Chem. Phys., 2005, 123, 204713.

19 H. Ikari, K Okanishi, M. Tomita and T. Ishidate, Opt. Mater., 2008, 30, 1323-1326. 
20 E. M. Purcell, Phys. Rev., 1946, 69, 681.

21 K. J. Vahala, Nature, 2003, 424, 839-846.

22 K. C. Ng, W. B. Whitten, S. Arnold and J. M. Ramsey, Anal. Chem., 1992, 64, 2914.

23 L. M. Folan, S. Arnold and S. D. Druger, Chem. Phys. Lett., 1985, 118, 322-327.

24 H. Fujiwara, K. Sasaki and H. Masuhara, ChemPhysChem, 2005, 6, 2410-2416.

25 S. Arnold, J. Chem. Phys., 1997, 106, 8280-8282.

26 D. V. O’Connor and D. Phillips, Time-Correlated Single Photon Counting (Academic Press, New York, 1986).

27 P. W. Barber and S. C. Hill, Light Scattering by Particles: Computational Methods (World Scientific, Singapore, 1990), chap. 4.

28 The refractive index of an aqueous sodium chloride $\left(1.0 \mathrm{~mol} / \mathrm{dm}^{3}\right)$ solution was calculated to be 1.343 according to the data published in W. A. Roth and K. Scheel, Landolt-Börnstein Physikalisch-chemische Tabellen (Springer-Verlag, Berlin Heidelberg, 1923).

29 N. Kitamura, M. Yamada, S. Ishizaka and K. Konno, Anal. Chem., 2005, 77, 6055-6061.

30 H. Yokoyama and S. D. Brorson, J. Appl. Phys., 1989, 66, 4801-4805. 
Table 1. Emission decay parameters of Ru(bpy $)_{3}{ }^{2+}$ in a water droplet and in an aqueous Solution.

\begin{tabular}{ccccccc}
\hline $\begin{array}{c}\text { Diameter } \\
/ \mu \mathrm{m}\end{array}$ & $\tau_{1} / \mathrm{ns}$ & $A_{1}$ & $\tau_{2} / \mathrm{ns}$ & $A_{2}$ & $\chi^{2 \mathrm{a}}$ & $\mathrm{DW}^{\mathrm{a})}$ \\
\hline 3.0 & $28.8 \pm 0.2$ & 0.95 & $403 \pm 4$ & 0.05 & 1.15 & 1.88 \\
3.3 & $31.2 \pm 0.2$ & 0.96 & $403 \pm 4$ & 0.04 & 1.06 & 1.79 \\
3.9 & $40.5 \pm 0.2$ & 0.93 & $403 \pm 4$ & 0.07 & 1.06 & 1.77 \\
4.0 & $44.1 \pm 0.2$ & 0.95 & $403 \pm 5$ & 0.05 & 1.07 & 1.95 \\
4.3 & $58.4 \pm 0.3$ & 0.89 & $403 \pm 4$ & 0.11 & 1.04 & 1.92 \\
4.6 & $82.7 \pm 0.3$ & 0.95 & $403 \pm 3$ & 0.05 & 1.04 & 1.88 \\
4.8 & $98.8 \pm 0.3$ & 0.94 & $403 \pm 7$ & 0.06 & 1.12 & 1.96 \\
5.0 & $141 \pm 0.6$ & 0.88 & $403 \pm 10$ & 0.12 & 1.06 & 1.99 \\
6.0 & $256 \pm 5.6$ & 0.77 & $403 \pm 27$ & 0.23 & 1.03 & 1.83 \\
& & & & & & \\
Bulk & & & $403 \pm 1$ & 1.00 & 1.07 & 2.01 \\
\hline
\end{tabular}

a) $\chi^{2}$ and DW represent the $\chi^{2}$ and Durbin-Watson parameters for the fitting, respectively. 
Table 2. Fitting parameters for the emission decay profiles in Figure 6.

\begin{tabular}{ccccccc}
\hline Index & $\begin{array}{c}I_{\mathrm{ex}}^{\mathrm{a}} \\
\mathrm{pJ} / \mathrm{pulse}\end{array}$ & $\begin{array}{c}\text { Diameter } \\
/ \mu \mathrm{m}\end{array}$ & $\tau_{1} / \mathrm{ns}$ & $A_{1}$ & $\tau_{2} / \mathrm{ns}$ & $A_{2}$ \\
\hline (a) & 7 & 4.7 & $97.1 \pm 0.6$ & 0.93 & $405 \pm 11$ & 0.07 \\
(b) & 3 & 4.6 & $82.0 \pm 0.5$ & 0.94 & $406 \pm 11$ & 0.06 \\
(c) & 13 & 4.3 & $57.7 \pm 0.3$ & 0.88 & $405 \pm 2$ & 0.12 \\
\hline
\end{tabular}

a) $I_{\mathrm{ex}}$ represents the excitation laser pulse energy before introducing the beam to the objective lens. 


\section{Figure Captions}

Fig. 1 Schematic illustration of laser trapping and time-resolved spectroscopy system.

Fig. 2 Single micrometer-sized water droplet levitated in air by means of laser trapping technique.

Fig. 3 Emission spectra of $\mathrm{Ru}(\mathrm{bpy})_{3}{ }^{2+}$ in a bulk aqueous solution (a), in water droplets with diameters 6 (b), 7 (c), and $11 \mu \mathrm{m}(\mathrm{d})$, respectively.

Fig. 4 Panel (a) shows the emission spectrum of $\mathrm{Ru}(\mathrm{bpy})_{3}{ }^{2+}$ in a water droplet of radius $\sim 3.5 \mu \mathrm{m}$. Panel (b) shows the theoretical simulation of the scattering efficiency $\left(Q_{\mathrm{s}}\right)$ of a droplet of refractive index 1.343 and radius $3.373 \mu \mathrm{m}$. Dashed lines are guides to eyes.

Fig. 5 Emission decay profiles of $\mathrm{Ru}(\mathrm{bpy})_{3}{ }^{2+}$ observed at $620 \pm 6 \mathrm{~nm}$ in a bulk aqueous solution (a) and in aerosol water droplets with diameters 5 (b), 4 (c), and $3 \mu \mathrm{m}$ (d), respectively. The red line represents the best fitting for each single- or double-exponential function.

Fig. 6 Emission decay profiles of $\mathrm{Ru}(\mathrm{bpy})_{3}{ }^{2+}$ observed at $620 \pm 6 \mathrm{~nm}$ in an aerosol water droplet with the excitation laser pulse energies at 7 (a), 3 (b), and 13 pJ/pulse (c), respectively. The diameter of the droplet gradually decreased from $4.7 \mu \mathrm{m}$ (a) to 4.6 (b) or $4.3 \mu \mathrm{m}$ (c), owing to evaporation of water. The red lines represent the best fittings by double-exponential functions. 
Fig. 7 Comparison between experimentally and theoretically determined enhancement factors. The solid squares are ratios of the fast decay rates to the slow ones calculated from the lifetimes in Table 1. The dashed and solid curves represent the particle-size dependence of the enhancement factor estimated from eqns (5) and (7), respectively. 


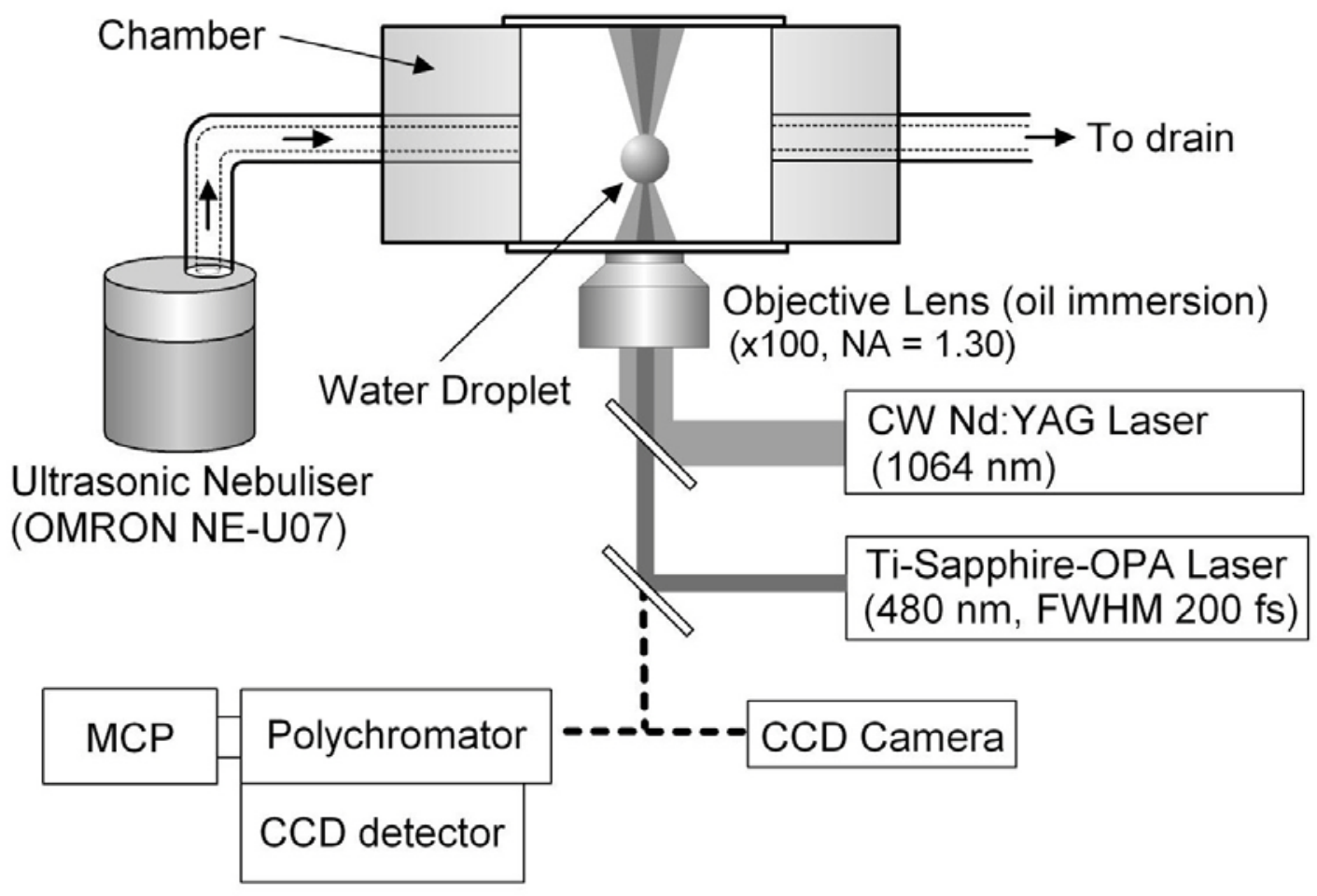

Fig. 1 S. Ishizaka et al. 
$10 \mu \mathrm{m}$

Fig. 2 S. Ishizaka et d. 


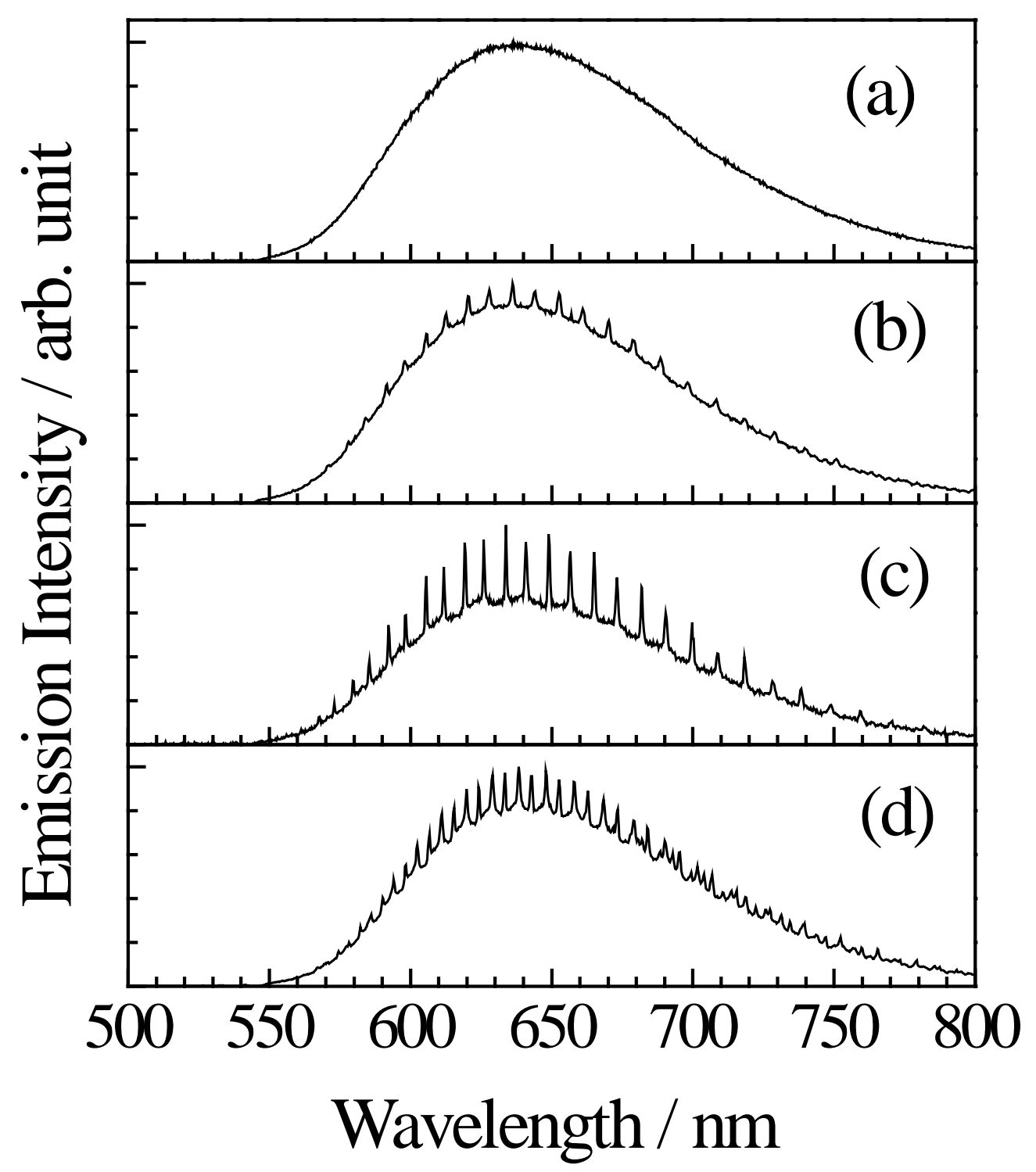

Fig. 3 S. Ishizaka et al. 


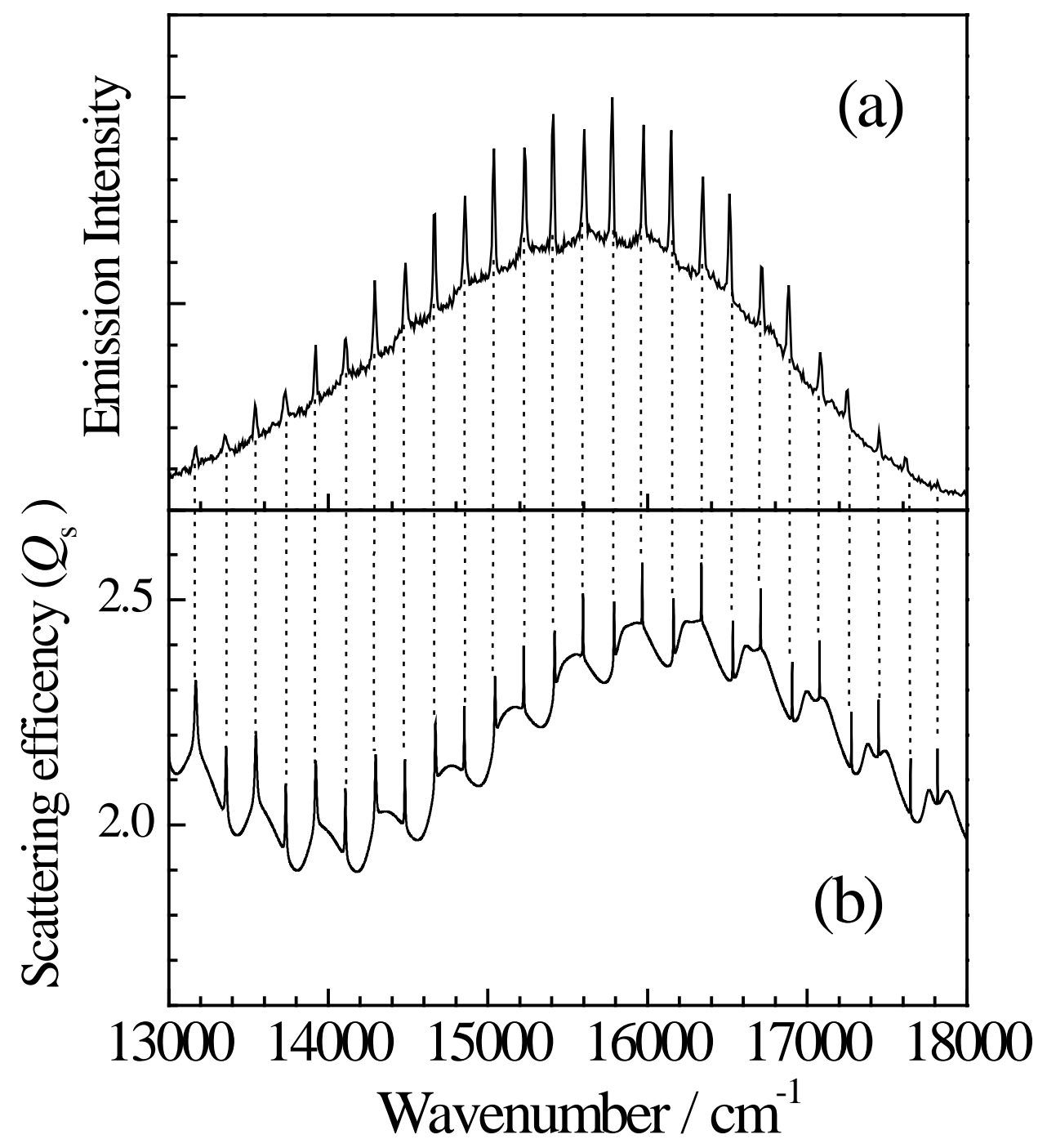

Fig. 4 S. Ishizaka et al. 


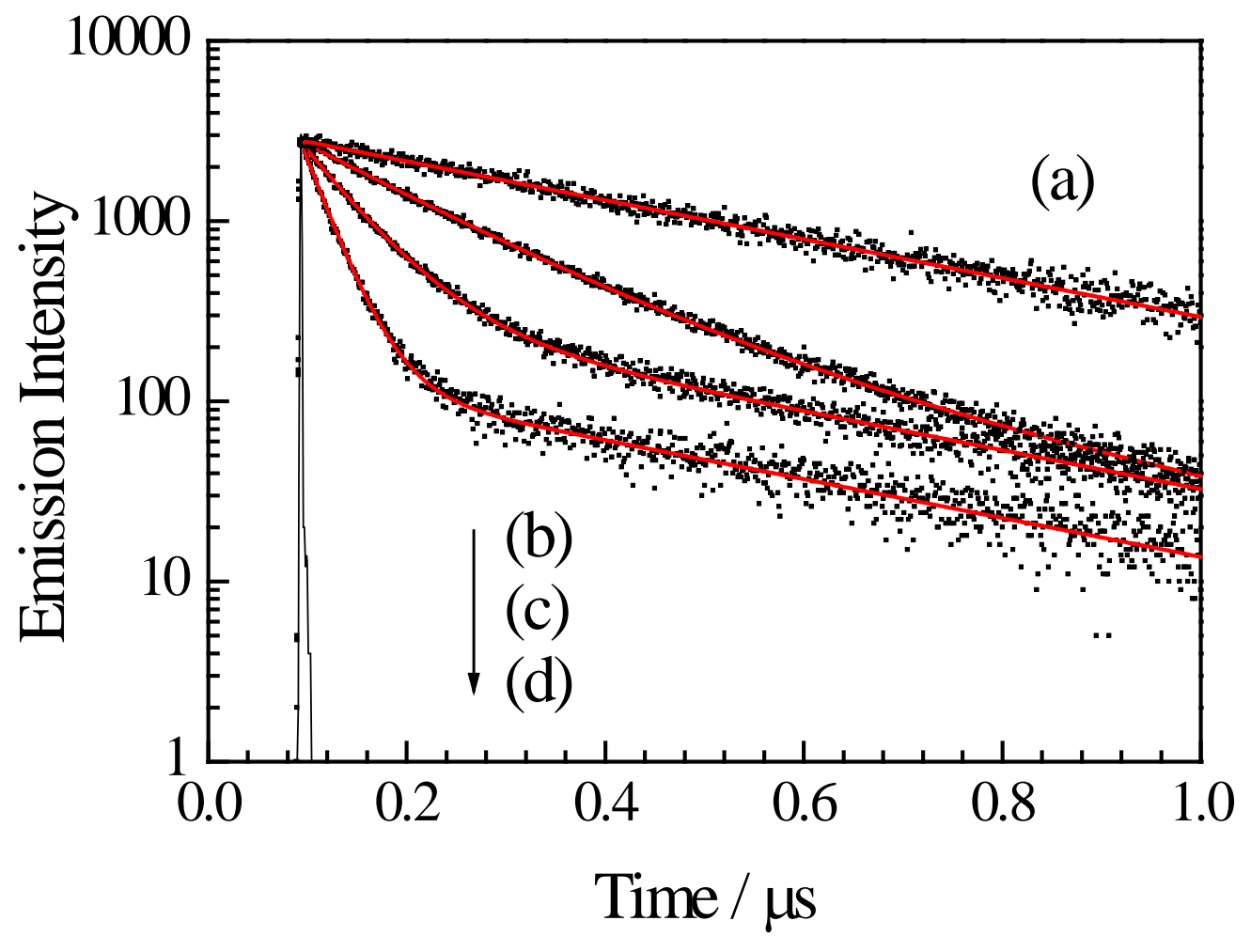

Fig. 5 S. Ishizaka et al. 


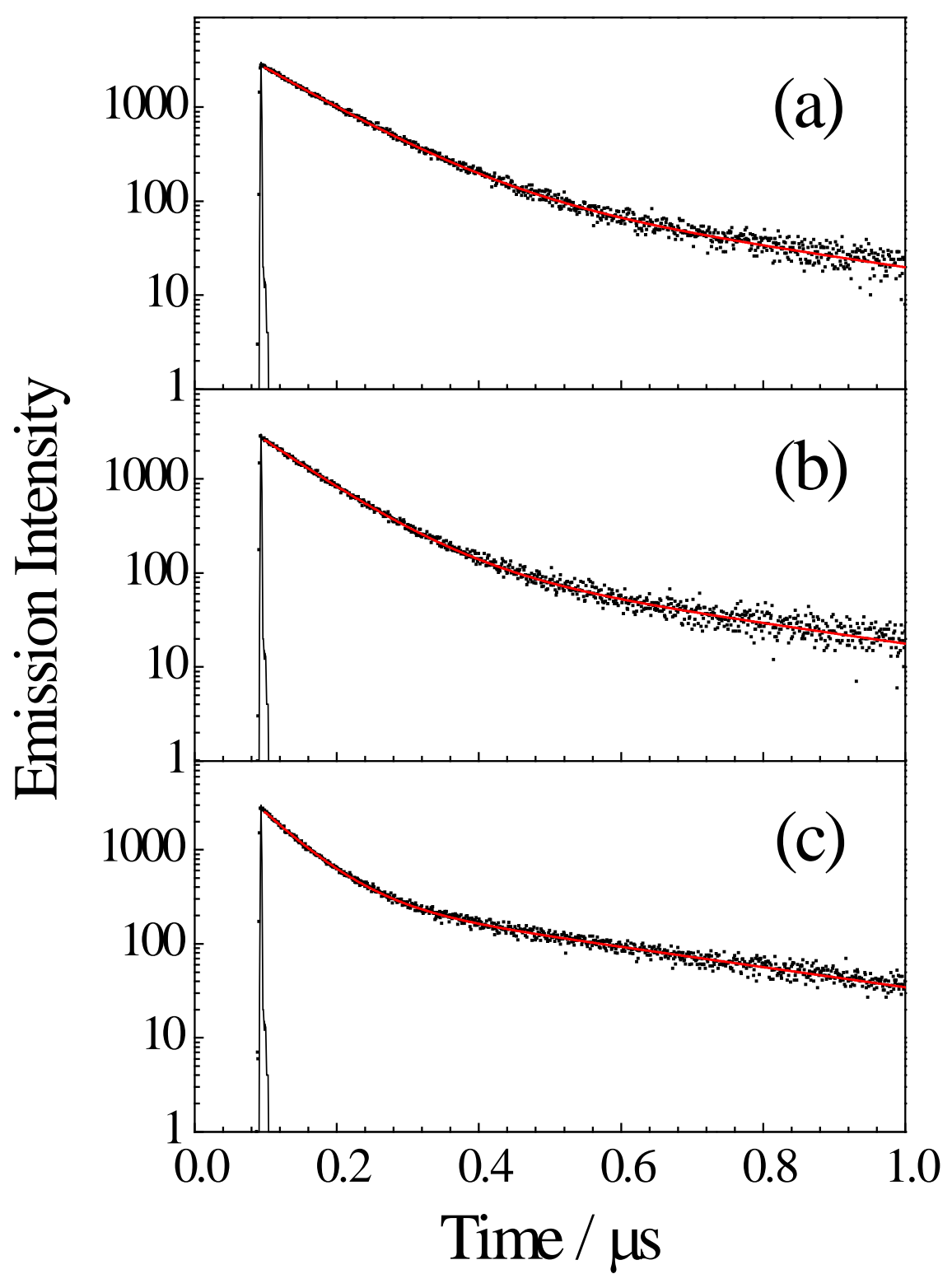

Fig. 6 S. Ishizaka et al. 


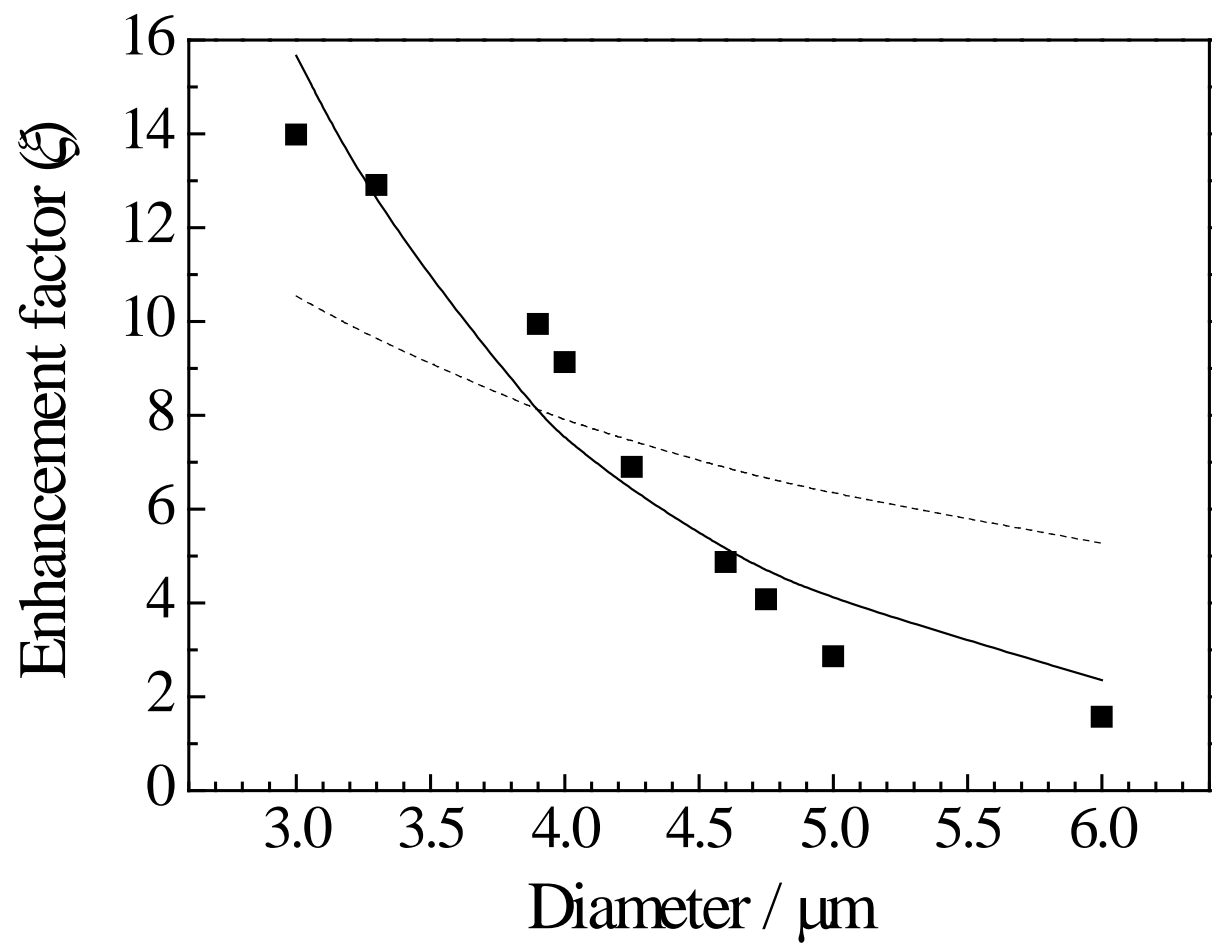

Fig. 7 S. Ishizaka et al. 